\title{
On the Pre-impact Orbital Evolution of 2018 LA, Parent Body of the Bright Fireball Observed over Botswana on 2018 June 2
}

\author{
Carlos de la Fuente $\operatorname{Marcos}^{1}$ and Raúl de la Fuente Marcos ${ }^{2}$ \\ ${ }^{1}$ Universidad Complutense de Madrid \\ Ciudad Universitaria, E-28040 Madrid, Spain \\ ${ }^{2}$ AEGORA Research Group \\ Facultad de Ciencias Matemáticas \\ Universidad Complutense de Madrid \\ Ciudad Universitaria, E-28040 Madrid, Spain
}

Keywords: minor planets, asteroids: general — minor planets, asteroids: individual (454100, 2016 LR, $\left.2018 \mathrm{BA}_{5}, 2018 \mathrm{LA}\right)$ 
On 2018 June 2, meteoroid 2018 LA became the third natural body ever to be observed before entering our atmosphere; ${ }^{1}$ similarly small asteroids 2014 AA and 2008 TC $_{3}$ had stricken the Earth on 2014 January 2 (de la Fuente Marcos et al. 2016; Farnocchia et al. 2016) and 2008 October 7 (Jenniskens et al. 2009; Oszkiewicz et al. 2012), respectively. Asteroid $2008 \mathrm{TC}_{3}$ disintegrated as a superbolide over northern Sudan (Jenniskens et al. 2009), 19 hours after discovery, and 2018 LA completely broke up in a fireball over South Africa and Botswana, over 8 hours after being first observed. ${ }^{2}$ Meteoroid 2014 AA was not visually observed to disintegrate, but infrasound recordings showed that it exploded over the Atlantic Ocean less than a day after being first spotted (de la Fuente Marcos et al. 2016; Farnocchia et al. 2016). Infrasound sensors also pinpointed the impact location of 2018 LA. The three asteroids had similar sizes of a few meters; such small bodies are probably fragments of larger objects. The study of the orbital evolution of such fragments prior to impact can help in understanding how asteroid disruptions take place (Trigo-Rodríguez et al. 2007).

The current orbit determination of 2018 LA (epoch JD 2458200.5, 23-March-2018, solution date 5-June-2018) is based on 14 observations for a data-arc span of $3.78 \mathrm{~h}$ and has semimajor axis, $a=1.374 \pm 0.002$ au, eccentricity, $e=0.4303 \pm 0.0009$, inclination, $i=4.284 \pm 0$.004, longitude of the ascending node, $\Omega=71.8795 \pm 0.0013$, and argument of perihelion, $\omega=256^{\circ} .04 \pm 0.03$; with an absolute magnitude of $30.6 \pm 0.3$, it may have been $2-5 \mathrm{~m}$ wide. ${ }^{3}$ This orbit determination is used to investigate the possible presence of known near-Earth objects (NEOs) moving in similar orbits, following the approach discussed by de la Fuente Marcos et al. $(2015,2016)$ and applying the $D$-criteria. We found several objects with values of the $D$-criteria with respect to 2018 LA under 0.05 and here we focus on three of them: (454100) $2013 \mathrm{BO}_{73}(a=1.33192556 \pm 0.00000002 \mathrm{au}, e=0.41841228 \pm 0.00000007, i=4.543978 \pm 0.000007$, $\left.D_{\mathrm{R}}=0.024\right), 2016 \mathrm{LR}\left(a=1.3813 \pm 0.0009 \mathrm{au}, e=0.4351 \pm 0.0005, i=2.542 \pm 0.002, D_{\mathrm{R}}=0.0055\right)$, and $2018 \mathrm{BA}_{5}$ $\left(a=1.3670 \pm 0.0009 \mathrm{au}, e=0.4310 \pm 0.0005, i=4.539 \pm 0.007, D_{\mathrm{R}}=0.010\right)$. The orbit of 2018 BA $\mathrm{A}_{5}$ is particularly close to that of $2018 \mathrm{LA}$ in terms of $a, e$, and $i$. Our results are based on the latest orbit determinations available from JPL's Small-Body Database and our $N$-body simulations were performed as described by de la Fuente Marcos et al. (2015, 2016). These four objects exhibit rather chaotic orbital evolutions and experience flybys with Venus, the Earth-Moon system, and Mars.

Figure 1 shows the pre-impact orbital evolution of 2018 LA and the three NEOs moving along relatively similar orbits. All of them encounter the Earth at distances close to or below the Hill radius (top panel). The evolution in $a$ (second to top panel) and $e$ (second to bottom panel) is somewhat alike (at least during part of the simulated time), but that of $i$ (bottom panel) is less correlated. These objects cannot be the result of a recent fragmentation event, but they could be part of a dynamical grouping (de la Fuente Marcos \& de la Fuente Marcos 2016).

In this Note, we have explored the pre-impact orbital evolution of 2018 LA, the parent body of the fireball observed over South Africa and Botswana on 2018 June 2. We have identified a few known NEOs with orbits similar to that of the impactor. Although the overall dynamical evolution of these NEOs bears some resemblance, it cannot be concluded that any of them might have been physically related to each other in the relatively recent past (see Figure 1). On the other hand, three other bolides were observed early in June: Crete on 2002 June 6, Washington State on 2004 June 3, and Reisadalen on 2007 June 7 (de la Fuente Marcos \& de la Fuente Marcos 2015). It is unclear whether these events could be related to each other. An even brighter superbolide was observed over Botswana on 2009 November 21 (de la Fuente Marcos \& de la Fuente Marcos 2015).

We thank S. J. Aarseth for providing the code used in this research and A. I. Gómez de Castro for providing access to computing facilities. This work was partially supported by the Spanish MINECO under grant ESP2015-68908-R. In preparation of this Note, we made use of the NASA Astrophysics Data System and the MPC data server.

\section{REFERENCES}

1 MPEC 2018-L04: 2018 LA

2 Fireball and Bolide Data

3 JPL's Small-Body Database de la Fuente Marcos, C., \& de la Fuente Marcos, R. 2015,

MNRAS, 446, L31

de la Fuente Marcos, C., \& de la Fuente Marcos, R. 2016, MNRAS, 456, 2946 


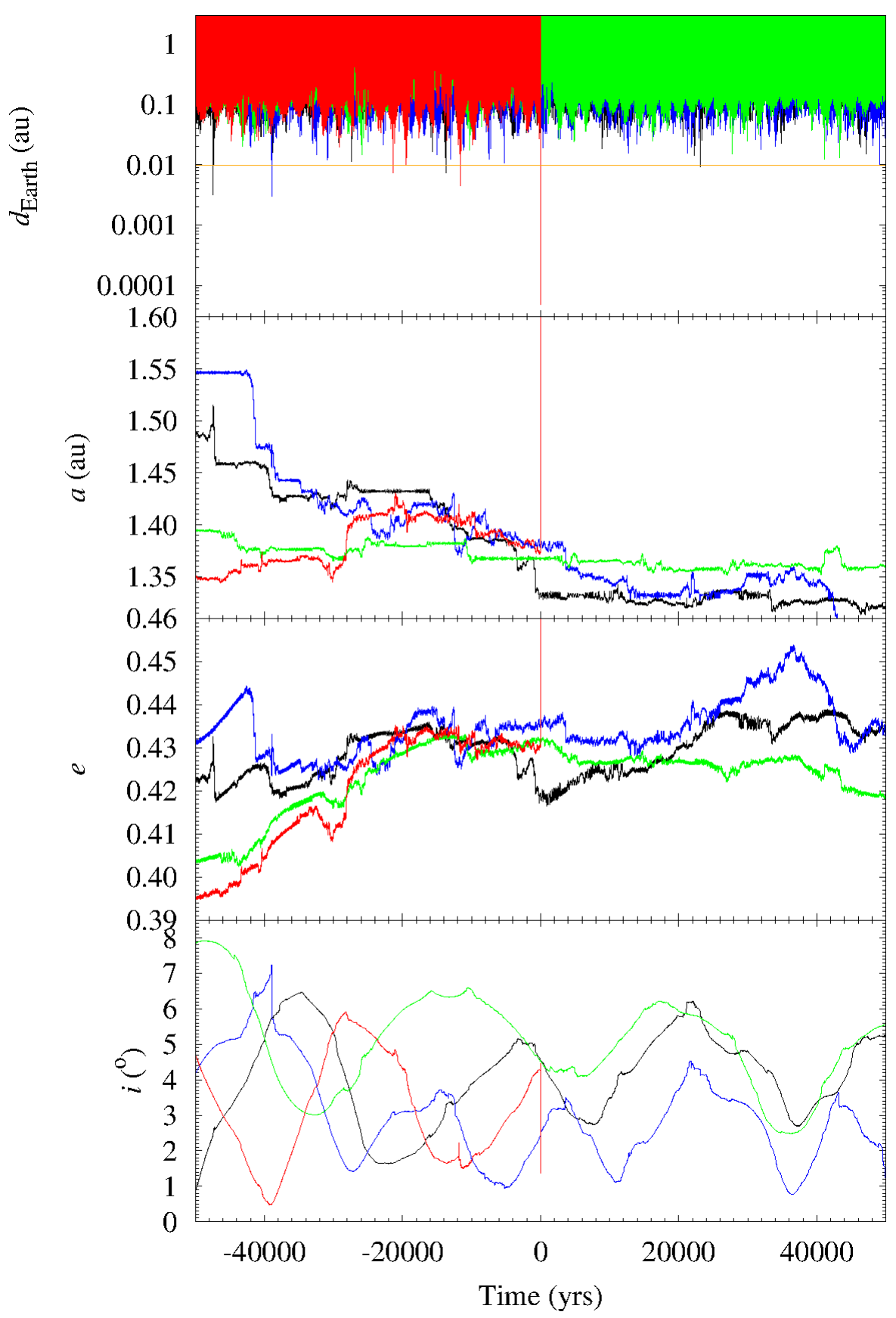

Figure 1. Evolution of the values of the perigee distance (top panel) - the Hill radius of the Earth is 0.0098 au (orange) semimajor axis (second to top panel), eccentricity (second to bottom panel), and inclination (bottom panel) of the nominal orbits (zero instant of time, epoch JD 2458200.5 TDB, 23-March-2018) of (454100) $2013 \mathrm{BO}_{73}$ (black), $2016 \mathrm{LR}$ (blue), $2018 \mathrm{BA} 5$ (green), and 2018 LA (red). Our calculations show that 2018 LA collided with our planet on JD 2458272.20259 TDB, JPL's Small-Body Database shows as impact time JD 2458272.19814 TDB.

de la Fuente Marcos, C., de la Fuente Marcos, R., \&

Aarseth, S. J. 2015, ApJ, 812, 26

de la Fuente Marcos, C., de la Fuente Marcos, R., \& Mialle,

P. 2016, Ap\&SS, 361, 358

Farnocchia, D., Chesley, S. R., Brown, P. G., \& Chodas,

P. W. 2016, Icarus, 274, 327
Jenniskens, P., Shaddad, M. H., Numan, D., et al. 2009, Nature, 458, 485

Oszkiewicz, D., Muinonen, K., Virtanen, J., Granvik, M., \& Bowell, E. 2012, Planet. Space Sci., 73, 30

Trigo-Rodríguez, J. M., Lyytinen, E., Jones, D. C., et al. 2007, MNRAS, 382, 1933 